\title{
Use of quantitative diffusion-weighted MR imaging (DWI) in differentiating between glioblastoma and primary central nervous system lymphoma in real-time exam interpretation
} \author{
$\mathrm{Cai}^{5}$ and Arash Kamali ${ }^{1 *}$ \\ ${ }^{1}$ Department of Diagnostic and Interventional Imaging, University of Texas Health Science Center at Houston, Texas, USA \\ ${ }^{2}$ McGovern Medical School, University of Texas Health Science Center at Houston, Texas, USA \\ ${ }^{3}$ Memorial Hermann Hospital, TMC, Texas, USA \\ ${ }^{4}$ Vivian L. Smith Department of Neurosurgery, University of Texas Health Science Center at Houston, Texas, USA \\ ${ }^{5}$ Department of Internal Medicine, University of Texas Health Science Center at Houston, Texas, USA
}

Christopher Stuart ${ }^{1}$, Pejman Rabiei ${ }^{1}$, Andrea Lugo ${ }^{2}$, Octavio Arevalo ${ }^{1}$, Laura Ocasio ${ }^{3}$, Mumtaz Syed ${ }^{1}$, Roy Riascos ${ }^{1}$, Jay-Jiguang Zhu ${ }^{4}$, Chunyan

\begin{abstract}
Given the significant different treatment strategy of glioblastoma compared to lymphoma, early non-invasive differentiation of these two malignant brain tumours is essential for treatment planning. Our study investigates the feasibility of differentiation of glioblastoma from lymphoma by measuring the apparent diffusion coefficient (ADC) values of the tumour using a very easy method on radiology PACS workstation. Forty-two (42) patients with pathology proven glioblastoma and 30 patients with pathology proven primary CNS lymphoma were retrospectively reviewed. Two different readers placed the regions of interest in the nonhaemorrhagic darkest solid area of the tumour to measure the ADC values. The median of the "Mean ADC Values" (averaged between two interpreters) in the primary CNS lymphoma group was significantly lower than the median of "Mean ADC Values" in the glioblastoma group for both readers $(p<0.0001)$ [582 $(511,687) \times 10^{-6} \mathrm{~mm}^{2} / \mathrm{s}$ for the lymphoma group and $789(734,896) \times 10^{-6} \mathrm{~mm}^{2} / \mathrm{s}$ for the GBM group]. Our study offers a novel, effective and easy approach for differentiation of the glioblastoma from lymphoma.
\end{abstract}

\begin{abstract}
Abbreviations: DWI: Diffusion Weighted Imaging; ADC: Apparent Diffusion Coefficient; CNS: Central Nervous System; GMB: Glioblastoma; PACS: Picture Archiving and Communication System; FLAIR: Fluid Attenuation Inversion Recovery; T1WI: T1 Weighted Image; T2WI: T2 Weighted Image; GRE: Gradient Echo; SWI: Susceptibility Weighted Imaging; ROI: Region Of Interest; DSC: Dynamic Susceptibility-Weighted Imaging; DCE: Dynamic Contrast Enhancement; MR: Magnetic Resonance; rCBV: Relative Cerebral Blood Volume.
\end{abstract}

\section{Introduction}

Glioblastoma (GBM) has been established as the most common malignant brain tumour in adults. GBM is characterized by fast growth and poor prognosis [1]. Median survival is generally less than one year from the time of diagnosis, and even in the most favourable situations, most patients will die within two years [2]. Although less common than GBM, the incidence of primary central nervous system (CNS) lymphoma has increased in the last three decades, especially in males [3]. Primary CNS lymphoma usually manifests as a highgrade non-Hodgkin's B-cell lymphoma with aggressive features. The median 5-year survival for primary CNS lymphoma is around $30 \%$. The treatment options for GBM and primary CNS lymphoma are significantly different. In GBM the first-line treatment is surgical resection [4]. Whereas, in primary CNS lymphoma, the first-line treatment is chemotherapy $[5,6]$. Consequently, these differences highlight the need to distinguish between these two entities on early diagnostic imaging.

Diffusion weighted imaging takes advantage of the diffusion of water molecules in normal human tissues and can create images where the signal is dependent on this molecular (Brownian) motion. The apparent diffusion coefficient (ADC) can then be calculated from these images and provides useful diagnostic information. Lower ADC values denote a higher degree of restricted diffusion, and these values can be used to quantitatively assess differences in the degree of restricted diffusion [7]. Previous investigations by Sugahara et al. [7] and Kono et al. [8] have demonstrated lower grade gliomas will typically have higher ADC values. Whereas higher grade gliomas typically have lower $\mathrm{ADC}$ values indicative of restricted diffusion secondary to hypercellularity $[7,8]$. Multiple other studies have shown that primary CNS lymphoma demonstrates restricted diffusion associated with the tumour's hypercellularity [9-11].

${ }^{*}$ Correspondence to: Arash Kamali, Department of Diagnostic and Intervention Imaging, University of Texas Health Science Center, Houston, Texas, USA, Tel: 713-704-1704; Fax: 713-704-1715; E-mail: Arash.Kamali@uth.tmc.edu

Key words: glioblastoma, primary CNS lymphoma, mean ADC values

Received: May 02, 2019; Accepted: May 13, 2019; Published: May 15, 2019 
Stuart C (2019) Use of quantitative diffusion-weighted MR imaging (DWI) in differentiating between glioblastoma and primary central nervous system lymphoma in real-time exam interpretation

Previous investigations by Guo et al. [9] and Yamasaki et al. [11] have directly compared GBM and primary CNS lymphoma showing that differences in ADC value between primary CNS lymphoma and GBM are statistically significant - with lymphoma having lower ADC values. Yet, other studies have revealed this comparison to be more complicated with hypercellular GBMs also exhibiting restricted diffusion associated with low ADC values [12-16]. In 2008, studies done by Toh $\mathrm{CH}$ et al. [17] and Yamashita $\mathrm{K}$ et al. [18] have shown primary CNS lymphoma to have lower ADC values than GBM. To obtain the ADC values, Toh et al. [17] selected a single ROI placed in the center of solid enhancing tumour region, while Yamashita et al. [18] and Doskaliyev et al. [19] placed multiple ROIs spread throughout the entire tumour mass using various techniques. All of these authors used advanced imaging processing software for ROI placement and ADC value analysis. We set out to in investigate if similar results could be achieved with an experienced reader using a different technique by placing an ROI on a standard PACS workstation to obtain ADC values which would aid in diagnosis during real-time interpretation of the exam.

\section{Materials and methods}

Approval by the University of Texas and Memorial Hermann institutional review board was obtained. Forty-two (42) patients with pathology proven glioblastoma and 30 patients with pathology proven primary CNS lymphoma were retrospectively reviewed. The patients were selected from referrals to Memorial Hermann hospital at the Texas Medical Center from 2014-2017. All patients underwent preoperative MR imaging on 1.5 or $3 \mathrm{~T}$ magnets. Patients included in the study had not initiated steroids or other therapy prior to imaging. Patients with previous brain surgery or biopsy were also excluded. The minimum sequences required were ADC, FLAIR, T1WI, T1WIpostcontrast, GRE or SWI, and T2WI.

ADC values were acquired by two blinded readers (neuroradiology fellows) who placed regions of interest (ROIs) using a Centricity PACS workstation. The ROIs were placed in the areas of greatest hypointensity on the solid segment of the tumour using the ADC map excluding the areas with necrotic, cystic or haemorrhagic components by correlating with the other sequences such as the Gradient Echo (GRE), Susceptibility Weighted Imaging (SWI) and T2 weighted images (Figure 1). Both the "Minimum ADC Values" and "Mean ADC Values" were recorded for each patient. Control ROIs were placed in the normal contralateral white matter. Placement of one ROI in the solid segment of a tumour on the ADC map provides the reader with

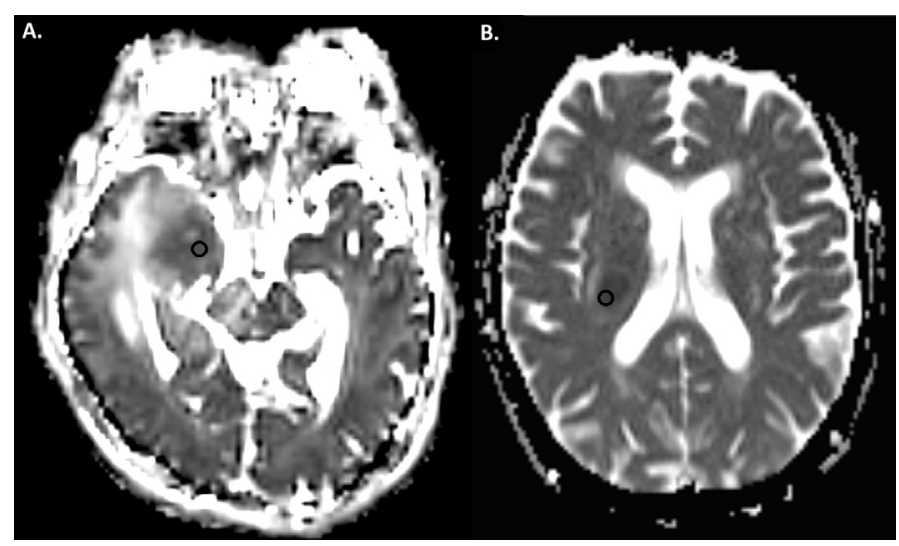

Figure 1. Apparent diffusion coefficient maps of a patient with Glioblastoma (A.) and a patient with primary CNS lymphoma (B.). Region of interests (black circles) are placed in the areas with greatest hypointensity on the solid segment of the tumor
3 numbers for that specific region: "Minimum ADC Value", "Mean ADC Value", and "Maximum ADC Value". Basically, a "Mean ADC Value" represents the mean of the ADC values of all the pixels/voxels in one region of interest. Previous studies have shown that "Minimum ADC Values" and "Mean ADC Values" are more reliable when they are implemented to distinguish different entities [9,11]. In this study and for simplification, we have only utilized the "Mean ADC values".

Given that the "Mean ADC Values" in both groups of patients showed non-normal distributions, we calculated the median of the "Mean ADC Values" for comparison.

Statistical analysis was performed with SAS 9.4 (SAS Institute Inc. Cary, NC). Age distribution between two groups was compared by two-sample t-test. Gender differences were compared by Chi-square test. The ADC value and ROI area differences between the two groups were compared by Wilcoxon Rank-Sum Test. A $p$-value less than 0.05 was considered significant.

\section{Results}

Demographics for GBM included 16 females and 26 males with an age range of 13-86 years with a mean age of 59.2. Demographic data for the primary CNS lymphoma group included 18 females and 12 males with an age range of 21-83 years and a mean age of 65 . Between the two groups there was no statistically significant difference in patient age ( $p=0.37)$ using the two-sample t-test. There was also no statistically significant difference in patient gender $(p=0.06)$ using the Chi-square test. The median ROI area for reader 1 was $21.7 \mathrm{~mm}^{2}$ and the median ROI area for reader 2 was $14.2 \mathrm{~mm}^{2}$ in the GBM group. The median areas for reader 1 and reader 2 in the lymphoma group were $27 \mathrm{~mm}^{2}$ and $13.5 \mathrm{~mm}^{2}$ respectively. The difference in area between the readers was statistically significant. However, it did not impact the results as there was no statistically significant difference in ROI area between the GBM and lymphoma groups.

The median of "Mean ADC Values" in the GBM group was 811 $(705,904) \times 10^{-6} \mathrm{~mm}^{2} / \mathrm{s}$ (Median (Q1, Q3). Q1:1st quartile; Q3:3rd quartile) for reader 1 and $789(758,881) \times 10^{-6} \mathrm{~mm}^{2} / \mathrm{s}$ for reader 2 . The median of "Mean ADC Values" in the lymphoma group was 599 $(510,718) \times 10^{-6} \mathrm{~mm}^{2} / \mathrm{s}$ for reader 1 and $589(508,654) \times 10^{-6} \mathrm{~mm}^{2} / \mathrm{s}$ for reader 2 (Figures 2,3 and Table 1 ).

The median of "Mean ADC Values" (averaged between two interpreters) in the primary CNS lymphoma group was significantly lower than the median of "Mean ADC Values" in the glioblastoma group $(p<0.0001)$, where they were $582(511,687) \times 10^{-6} \mathrm{~mm}^{2} / \mathrm{s}$ for the lymphoma group and $789(734,896) \times 10^{-6} \mathrm{~mm}^{2} / \mathrm{s}$ for the GBM group.

\section{Discussion}

We demonstrated that the median of "Mean ADC Values" is significantly different when comparing GBM and primary CNS lymphoma. Specifically, primary CNS lymphoma showed a lower median "Mean ADC Value" than GBM, a finding thought to be related to hyper viscosity of tissue secondary to hypercellularity. The inverse relationship between ADC values and hypercellularity is supported by previous investigations [9].

In our study the intra-tumoral focus with the greatest degree of restricted diffusion underwent quantitative $A D C$ value measurement by ROI placement. This novel approach does introduce a degree of subjectivity as the region is identified by the reader. However, it remains clinically pragmatic as the ROI can be placed at a standard 
Stuart C (2019) Use of quantitative diffusion-weighted MR imaging (DWI) in differentiating between glioblastoma and primary central nervous system lymphoma in real-time exam interpretation

Table 1. Comparison of intra-tumoral ADC values $\left(\times 10^{-6} \mathrm{~mm}^{2} / \mathrm{s}\right)$ between primary CNS lymphoma and Glioblastoma. (*Wilcoxon rank-sum test)

\begin{tabular}{|c|c|c|c|}
\hline \multicolumn{2}{|c|}{ Median (Q1, Q3) } & Primary CNS Lymphoma & Glioblastoma \\
\hline Reader 1 & "Mean ADC Value" & $599(510-718)$ & $811(705-904)$ \\
\hline Reader 2 & "Mean ADC Value" & $589(508-654)$ & $789(758-881)$ \\
\hline Average of 2 reads & "Mean ADC Value" & $582(511-687)$ & $789(734-869)$ \\
\hline
\end{tabular}

Q1: $1^{\text {st }}$ quartile, Q3: $3^{\text {rd }}$ quartile

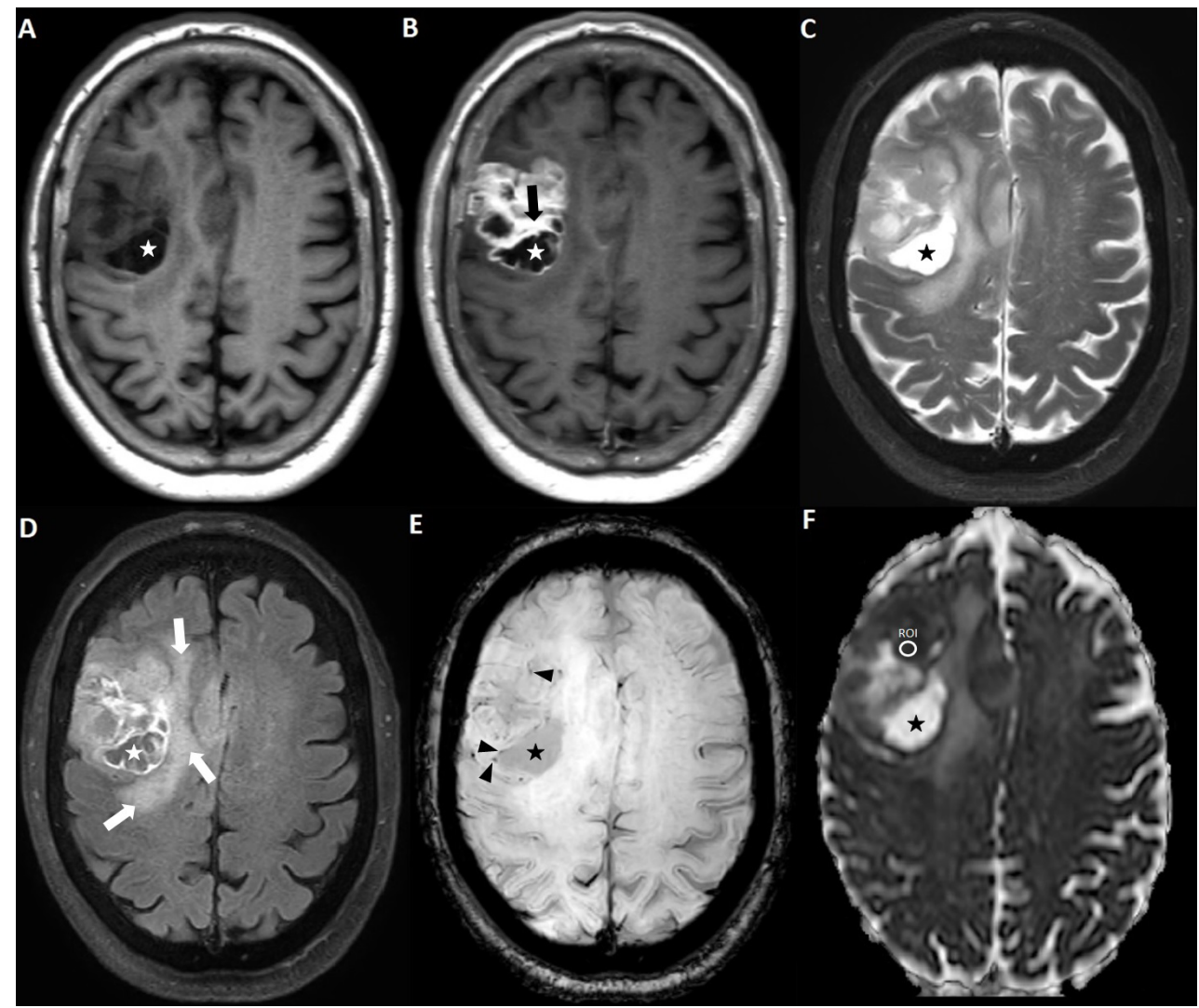

Figure 2. Axial magnetic resonance imaging of the brain including (A) T1WI, (B) post-contrast T1WI, (C) T2WI, (D) FLAIR, (E) SWI, and (F) ADC map. The area with greatest hypointensity on the ADC map is selected for ROI placement. Extra information derived from other MR sequences about cystic/necrotic regions (asterisks), enhancing regions (black arrow), vasogenic edema (white arrows), and spots of susceptibility (black arrowhead) is utilized for accurate ROI placement

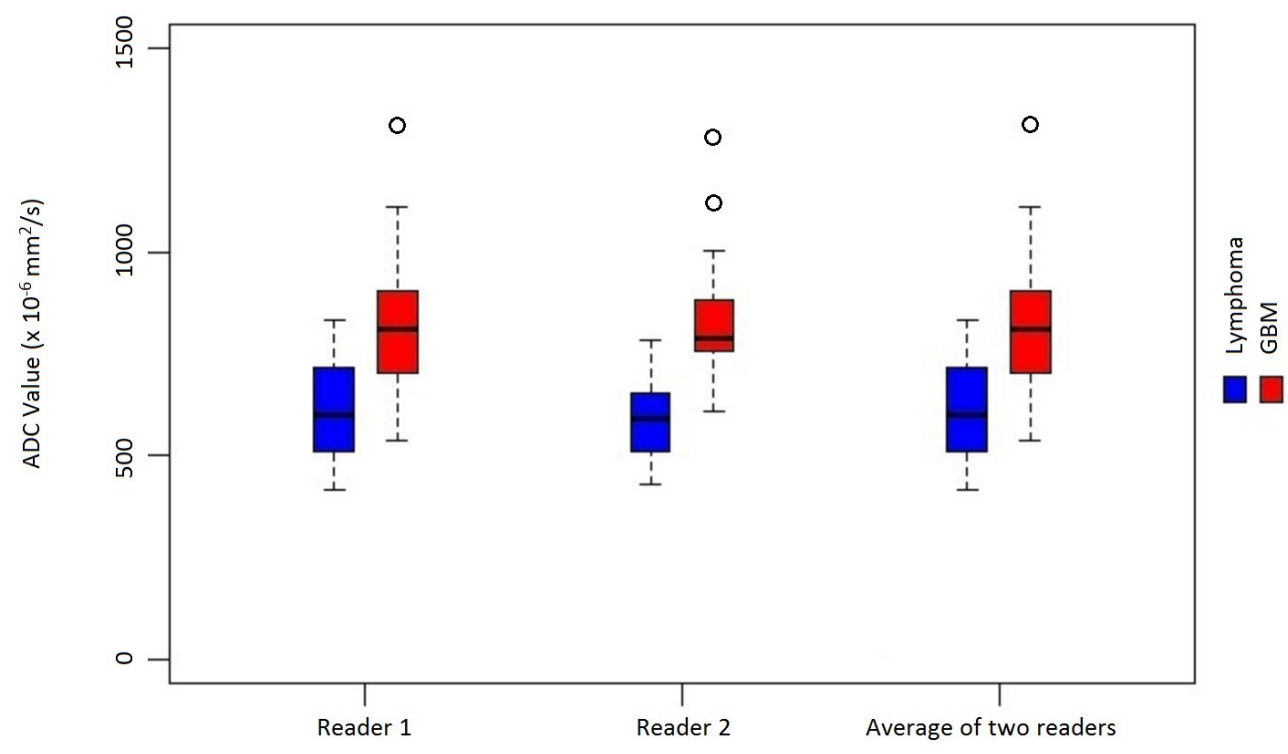

Figure 3. Distribution of "Mean ADC values" in primary CNS lymphoma and Glioblastoma 
Stuart C (2019) Use of quantitative diffusion-weighted MR imaging (DWI) in differentiating between glioblastoma and primary central nervous system lymphoma in real-time exam interpretation

PACS workstation while interpreting the exam using all available complementary sequences. It also doesn't burden the interpreter with placing multiple ROIs in different regions of the tumour which can be time consuming.

It remains necessary to ensure the ROI is placed in an area which excludes necrotic, cystic, or haemorrhagic components of the tumour. Haemorrhage or necrosis may show greater diffusion restriction than the hypercellular solid tumour due to the associated increased hyper viscosity. We also avoided measuring the cystic component of the tumour as this would cause a falsely elevated ADC value. The placement of the ROIs was without regard to the enhancing or nonenhancing component of the tumour since we aimed not to be biased by the enhancement of the tumour in our measurement. The rationale behind this was to include the non-enhancing components of the tumours specifically in GBM which is notorious for having non-enhancing solid component demonstrating FLAIR hyperintensity.

Our method produces data which validates previous findings which show that ADC values are lower in primary CNS lymphoma [19]. Median of "Mean ADC Values" in the glioblastoma group were significantly higher than the median of "Mean ADC Values" in the primary CNS lymphoma group $(p<0.0001)$, confirming the utility of using $\mathrm{ADC}$ value measurement in distinguishing these entities - even if the values are obtained from a standard PACS workstation. Our findings suggest that a "Mean ADC Value" less than $700 \times 10^{-6} \mathrm{~mm}^{2} / \mathrm{s}$ should lead you to favour primary CNS lymphoma in developing your differential diagnosis. Previous studies involved placing multiple ROIs while avoiding haemorrhagic and cystic components using conventional imaging and found similar results [19]. Ahn et al. [20] examined multiple ROI placement methods and found them to be inconsistent. They describe a technique that generates an ADC value from the entire tumour volume being the most reproducible, and supportive of the finding that primary CNS lymphoma has lower ADC values than glioblastoma. The study by Yamashita et al. [18] yielded similar results while excluding blood vessels, necrosis, haemorrhage, and calcification but involved placing $>10$ ROIs within an enhancing region of the tumour. The study by Toh et al. [17] examined ADC and FA values using Nordic ICE (Nordic Image Control and Evaluation Version 2.16; Nordic Imaging Lab, Bergen, Norway) with an ROI placed in the enhancing region and found a significant difference for both yielding similar results to our study.

Our approach is the easiest and more practical relative to prior studies which can facilitate the differentiation of these two important tumours faster and more efficiently. We measured ADC values in the region of greatest restricted diffusion present on the ADC map at a standard PACS workstation and this yielded statistically significant results. This confirms the validity of a more efficient method for measuring ADC values during real-time interpretation. However, an ADC value alone is not enough to provide a definite diagnosis when differentiating these tumours. An approach which uses ADC values complemented by other imaging techniques such as perfusion weighted imaging would provide the most accurate diagnosis to guide subsequent therapy. Kickingereder et al. [21] have shown that utilizing complementary conventional and advanced imaging techniques aids in differentiating GBM from primary CNS lymphoma. Dynamic susceptibility-weighted imaging (DSC), dynamic contrast enhancement (DCE), FDG-PET, and susceptibility-weighted imaging (SWI) have all been described to aid in differentiating GBM from primary CNS lymphoma [22-26]. Limitations in our study include sample size and selection bias since only patients who had pathological proven tumour were selected. In the near future, we will combine the ADC value data with $\mathrm{rCBV}$ perfusion ratios of the tumours for more robust and accurate non-invasive differentiation of primary CNS lymphoma and GBM.

In conclusion, an $\mathrm{ADC}$ value (a quantitative measure of restricted diffusion) is a useful imaging biomarker when differentiating between primary CNS lymphoma and GBM. A reproducible method to obtain $\mathrm{ADC}$ values during real-time interpretation is by placing a ROI over the solid areas of greatest restricted diffusion while avoiding necrotic and haemorrhagic components.

\section{Conclusion}

Quantitative diffusion-weighted MR imaging using ADC values in standard PACS software can increase diagnostic accuracy when attempting to differentiate glioblastoma and primary CNS lymphoma.

\section{References}

1. Stupp R, Mason WP, van den Bent MJ, Weller M, Fisher B, et al. (2005) Radiotherapy plus concomitant and adjuvant temozolomide for glioblastoma. $N$ Engl J Med 352: 987 996. [Crossref]

2. Buckner JC (2003) Factors influencing survival in high-grade gliomas. Semin Oncol 30:10-14. [Crossref]

3. Surawicz TS, McCarthy BJ, Kupelian V, Jukich PJ, Bruner JM, et al. (1999) Descriptive epidemiology of primary brain and CNS tumors: results from the Central Brain Tumor Registry of the United States, 1990-1994. Neuro Oncol 1: 14-25. [Crossref]

4. Giese A, Westphal M (2001) Treatment of malignant glioma: a problem beyond the margins of resection. J Cancer Res Clin Oncol 127: 217-225. [Crossref]

5. Deckert M, Engert A, Brück W, Ferreri AJ, Finke J, et al. (2011) Modern concepts in the biology, diagnosis, differential diagnosis and treatment of primary central nervous system lymphoma. Leukemia 25: 1797-1807. [Crossref]

6. Phillips EH, Fox CP, Cwynarski K (2014) Primary CNS Lymphoma. Curr Hematol Malig Rep 9: 243-253. [Crossref]

7. Sugahara T, Korogi Y, Kochi M, Ikushima I, Shigematu Y, et al. (1999) Usefulness of diffusion-weighted MRI with echo-planar technique in the evaluation of cellularity in gliomas. J Magn Reson Imaging 9: 53-60. [Crossref]

8. Kono K, Inoue Y, Nakayama K, Shakudo M, Morino M, et al. (2001) The role of diffusion-weighted imaging in patients with brain tumors. AJNR Am J Neuroradiol 22: 1081-1088. [Crossref]

9. Guo AC, Cummings TJ, Dash RC, Provenzale JM (2002) Lymphomas and high-grade astrocytomas: comparison of water diffusibility and histologic characteristics. Radiology 224: 177-183. [Crossref]

10. Stadnik TW, Chaskis C, Michotte A, Shabana WM, van Rompaey K, et al. (2001) Diffusion-weighted MR imaging of intracerebral masses: comparison with conventional MR imaging and histologic findings. AJNR Am J Neuroradiol 22: 969-976. [Crossref]

11. Yamasaki F, Kurisu K, Satoh K, Arita K, Sugiyama K, et al. (2005) Apparent diffusion coefficient of human brain tumors at MR imaging. Radiology 235: 985-991. [Crossref]

12. Chang YW, Yoon HK, Shin HJ, Roh HG, Cho JM (2003) MR imaging of glioblastoma in children: usefulness of diffusion/perfusion-weighted MRI and MR spectroscopy. Pediatr Radiol 33: 836-842. [Crossref]

13. Batra A, Tripathi RP (2004) Atypical diffusion-weighted magnetic resonance findings in glioblastoma multiforme. Australas Radiol 48: 388-391. [Crossref]

14. Hakyemez B, Erdogan C, Yildirim N, Parlak M (2005) Glioblastoma multiforme with atypical diffusion-weighted MR findings. Br J Radiol 78: 989-992. [Crossref]

15. Toh CH, Chen YL, Hsieh TC, Jung SM, Wong HF, et al. (2006) Glioblastoma multiforme with diffusionweighted magnetic resonance imaging characteristics mimicking primary brain lymphoma. Case report. J Neurosurg 105: 132-135. [Crossref]

16. Baehring JM, Bi WL, Bannykh S, Piepmeier JM, Fulbright RK (2007) Diffusion MRI in the early diagnosis of malignant glioma. J Neurooncol 82: 221-225. [Crossref]

17. Toh CH, Castillo M, Wong AM, Wei KC, Wong HF, et al. (2008) Primary cerebra lymphoma and glioblastoma multiforme: differences in diffusion characteristics evaluated with diffusion tensor imaging. AJNR Am J Neuroradiol 29: 471-475. [Crossref] 
Stuart C (2019) Use of quantitative diffusion-weighted MR imaging (DWI) in differentiating between glioblastoma and primary central nervous system lymphoma in real-time exam interpretation

18. Yamashita K, Yoshiura T, Hiwatashi A, Togao O, Yoshimoto K, et al. (2013) Differentiating primary CNS lymphoma from glioblastoma multiforme: assessment using arterial spin labeling, diffusion-weighted imaging, and ${ }^{18} \mathrm{~F}$-fluorodeoxyglucose positron emission tomography. Neuroradiology 55: 135-143. [Crossref]

19. Doskaliyev A, Yamasaki F, Ohtaki M, Kajiwara Y, Takeshima Y, et al. (2012) Lymphomas and glioblastomas: differences in the apparent diffusion coefficient evaluated with high b-value diffusion-weighted magnetic resonance imaging at 3T. Eur J Radiol 81: 339-344. [Crossref]

20. Ahn SJ, Shin HJ, Chang JH, Lee SK (2014) Differentiation between primary cerebral lymphoma and glioblastoma using the apparent diffusion coefficient: Comparison of three different ROI methods. PLoS One 9: e112948. [Crossref]

21. Kickingereder P, Wiestler B, Sahm F, Heiland S, Roethke M, et al. (2014) Primary Central Nervous System Lymphoma and Atypical Glioblastoma: Multiparametric Differentiation by Using Diffusion-, Perfusion-, and Susceptibility-weighted MR Imaging. Radiology 272: 843-850. [Crossref]
22. Kim HS, Jahng GH, Ryu CW, Kim SY (2009) Added value and diagnostic performance of intratumoral susceptibility signals in the differential diagnosis of solitary enhancing brain lesions: preliminary study. AJNR Am J Neuroradiol 30: 1574-1579. [Crossref]

23. Kickingereder P, Sahm F, Wiestler B, Roethke M, Heiland S, et al. (2014) Evaluation of microvascular permeability with dynamic contrast-enhanced MRI for the differentiation of primary CNS lymphoma and glioblastoma: radiologicpathologic correlation. AJNR Am J Neuroradiol 35: 1503-1508. [Crossref]

24. Liao W, Liu Y, Wang X, Jiang X, Tang B, et al. (2009) Differentiation of primary centra nervous system lymphoma and high-grade glioma with dynamic susceptibility contrastenhanced perfusion magnetic resonance imaging. Acta Radiol 50: 217-225. [Crossref]

25. Makino K, Hirai T, Nakamura H, Murakami R, Kitajima M, et al. (2011) Does adding FDG-PET to MRI improve the differentiation between primary cerebral lymphoma and glioblastoma? Observer performance study. Ann Nucl Med 25: 432-438. [Crossref]

26. Villano JL, Koshy M, Shaikh H, Dolecek TA, McCarthy BJ (2011) Age, gender, and racial differences in incidence and survival in primary CNS lymphoma. $\mathrm{Br} J$ Cancer 105: 1414-1418. [Crossref]

Copyright: (2019 Stuart C. This is an open-access article distributed under the terms of the Creative Commons Attribution License, which permits unrestricted use, distribution, and reproduction in any medium, provided the original author and source are credited. 